\title{
Structure Based Virtual Screening and Molecular Docking Studies for Identification of Allosteric Inhibitors against Zika Virus Protease NS2B-NS3
}

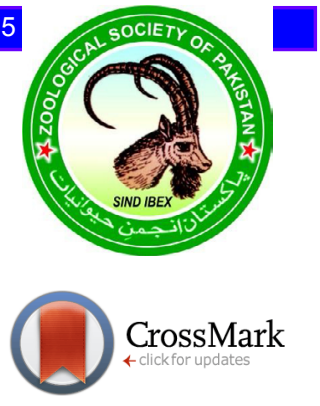

\author{
Musrrat Fatima, Muhammad Saad Khan, Hamid Rashid, Asim Mehmood, \\ Sumaira Kanwal, Muhammad Asif Rasheed and Farrukh Jamil* \\ Department of Biosciences, COMSATS University Islamabad, Sahiwal Campus, \\ Punjab
}

\section{A B S T RA C T}

Zika virus (ZIKV) has gained research interests after its recent outbreak in Brazil and America in 2015, where it affected millions of people. Studies have shown its role in developing microcephaly and GBS Syndrome. There is a need to develop drugs against ZIKV. Here we uses pharmacoinformatics techniques to identify potential inhibitors against ZIKV protease NS2B-NS3, which has a well-known role in the viral replication. By using three dimensional structure of the NS2B-NS3, an allosteric site has been identified in the protein. Moreover, the identified site is used for structure-based virtual screening. After screening 100,000 compounds, 14 compounds are selected, which fulfilled different already established drug likeliness parameters such as rule of five and nontoxic nature. These 14 compounds are used for pharmacophore modeling, and Zinc natural compounds database (ZND) is screened against the developed pharmacophore. The compounds with the highest pharmacophore fit scores are screened and docked on the identified allosteric site of NS2B-NS3. Analysis of the data showed two compounds (ZINC00845171, ZINC08782519) as potential inhibitors for NS2B-NS3 of ZIKV.

\begin{tabular}{l} 
Article Information \\
Received 12 March 2018 \\
Revised 15 April 2018 \\
Accepted 04 May 2018 \\
Available online 03 August 2018 \\
Authors' Contribution \\
FJ designed the study. MF, SK, \\
HR, AM and SK performed the \\
experiments and collected data. FJ \\
and MAR analyzed the data. FJ \\
gathered the data, supervised the \\
study and prepared the manuscript. \\
Key words \\
\hline Zika virus, NS2B-NS3, Protease, \\
Inhibitors, Virtual screening, Docking.
\end{tabular}

\section{INTRODUCTION}

Z ika virus (ZIKV) belongs to flavivirus genus. The other renowned members of the genus are: Dengue virus (DENV), West Nile virus, Japanese encephalitis virus and Yellow fever virus. ZIKV was isolated in 1947 from the serum of a monkey in Zika Forest (Dick et al., 1952). However, it emerged as a disease-causing agent in 2015. ZIKV was spread in Brazil in May 2015, and then it was rapidly spread throughout America (Campos et al., 2015). In 2016, 531,000 suspected cases of this virus were reported in different American territories. Moreover, a congenital syndrome has been associated with Zika virus (Ikejezie et al., 2017). The main vectors of this virus are Aedes aegypti mosquitoes, though other mosquito species can also contribute in its transmission (Colman et al., 2012). Additionally, several cases have been reported for sexual transfer of the virus (Ikejezie et al., 2017).

ZIKV genome is composed of, positive-sense, single-stranded RNA molecule of approximately $11 \mathrm{~kb}$ (Moghadam et al., 2016). Studies have shown that the

\footnotetext{
Corresponding author: farrukhccb@gmail.com; farrukh@ciitsahiwal.edu.pk 0030-9923/2018/0005-1709 \$ 9.00/0

Copyright 2018 Zoological Society of Pakistan
}

viral genome is translated into a single polyprotein, which is cleaved into 10 mature proteins by cellular and viral proteases. Studies have shown that 7 , out of these 10 , proteins are non-structural and designated NS1, NS2A, NS2B, NS3, NS4A, NS4B, and NS5. Remaining three are structural proteins including Capsid (C), Pre-membrane/ membrane (prM), and Envelope (E) proteins (Kuno and Chang, 2007; Baronti et al., 2014; Sironi et al., 2016).

ZIKV causes mild illness with symptoms fever, arthralgia, rash, conjunctivitis, muscle and joint pain (Tan et al., 2017). The current outbreak of ZIKV in Brazil and Latin America showed a positive correlation between viral infection and rise of microcephaly (Driggers et al., 2016). Moreover, studies have shown that ZIKV is also associated with neurological disorders such as GuillainBarre syndrome (Nowakowski et al., 2016).

Studies have highlighted NS2B-NS3, a serine protease, as a key player in ZIKV replication (Mackenzie, 2005; Li et al., 2017). The crystal structure of NS2BNS3 and substrate specificity profile has already been determined (Lei et al., 2016; Gruba et al., 2016). Analysis showed that the binding pocket of NS2B-NS3 protease is highly hydrophobic like the other similar proteases in other flavivirus (Yildiz et al., 2013). A recent study has targeted an allosteric site of NS2B-NS3 complex and they have identified certain compounds for the inhibition 
(hfNPS) of the protease (Shiryaev et al., 2017). However, the identified compounds are highly water soluble and host unfavorable pharmacokinetic parameters. The authors have suggested further study to identify inhibitors with favorable pharmacokinetic parameters (Shiryaev et al., 2017). In this study, we have screened 100,000 compounds for the inhibitors of NS2B-NS3, perform virtual screening against the allosteric site and determine naturally occurring compounds as inhibitor of the protease. It is a step toward developing a potential drug against ZIKV.

\section{METHODOLOGY}

\section{Allosteric site identification}

The allosteric site of DENV NS2B-NS3 protease (PDB: 2FOM) has already been reported (Mukhametov et al., 2014). By superposing ZIKV NS2B-NS3 (5GXJ) on the DENV NS2B-NS3 protease (PDB: 2FOM), we determined the allosteric site in ZIKV NS2B-NS3. Both protein structures are superimposed by using PyMol software (Lill and Danielson, 2011).

\section{Protein preparation for docking}

The NS2B-NS3 protein structure (PDB: 5GXJ) was retrieved from Protein Data Bank (https://www.rcsb. org). The chain B and water molecules were deleted using Chimera software for docking analysis. By using AutoDock Vina polar hydrogen and Kollman charges added in the structure (Trott and Olson, 2010). A grid with dimensions $100 \times 100 \times 100 \AA$ with center $\mathrm{x}=-0.254, \mathrm{y}=$ 11.832 and $\mathrm{z}=-24.015$ was used for targeted docking in the allosteric site of NS2B-NS3.

\section{Virtual screening}

Mcule was used to perform the structure-based virtual screening (SBVS) (Kiss et al., 2012). It uses a built-in AutoDock Vina tool to perform docking of small ligands against target site. Each ligand of the selected database is docked against the selected allosteric pocket. Ligands were scored according to the minimum binding energy.

\section{Drug-like properties determination of the selected compounds}

The best compounds were checked by OSIRIS property explorer (Actelion Pharmaceuticals Ltd., Allschwil, Switzerland) for drug-like properties. Molecular weight, LogP, hydrogen bond donor, hydrogen bond acceptor and polar surface area were determined. Those compounds that fulfill rule of five (Ro5) criteria were further filtered by checking the compound toxicity using Mcule lead optimization tool toxicity checker (Kiss et al., 2012). AdmetSar was used to check the ADMET (Absorption,
Distribution, Metabolism, Excretion, Toxicity) properties (Cheng et al., 2012), such as blood brain barrier, human intestinal absorption, caco-2 permeability, honey bee toxicity, fish toxicity etc.

\section{Pharmacophore modeling}

Ligands based pharmacophore modeling performed by Ligand scout (Wolber and Langer, 2005). Ligand scout is a tool that generates three dimensional pharmacophore models from structural data of macromolecules ligands complexes. Zinc Natural Derivatives (ZND) database downloaded from Zinc specialized databases. Each compound of the database is screened against the generated pharmacophore and scored according to matching features.

\section{Compound interactions and toxicity}

The compounds with highest pharmacophore fit score were further screened through their different physical properties such as Ro5, toxicity and ADMET parameters. The filtered compounds were docked to the allosteric sites of NS2B-NS3, and their interactions with the allosteric sites residues were checked by using LIGPLOT (Wallace et al., 1995).

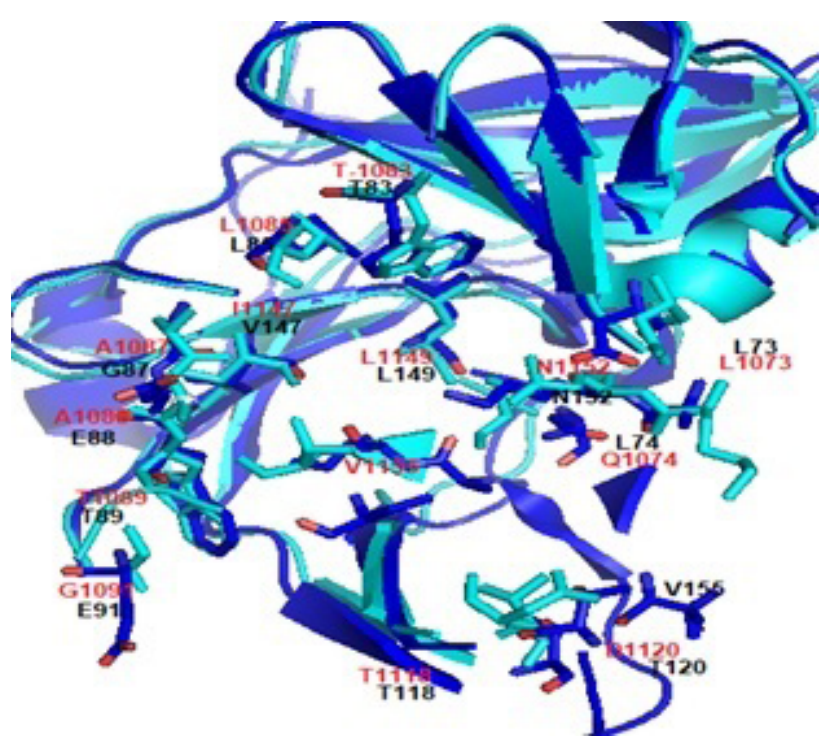

Fig. 1. Superimpose NS2B-NS3 of DENV (blue) on NS2B-NS3 of ZIKV (cyan). It also shows allosteric site residues of DENV (red) and ZIKV (black).

\section{RESULTS AND DISCUSSION}

In the post genome era, bioinformatics has emerged a valuable tool for large data analysis. Several applications of different bioinformatics tools have been reported ranging from gene prediction to drug identification (Mukhametov et al., 2014; Tariq et al., 2016; Rana et al., 2017; Jamil et 
al., 2018). In this study we focus to identify drug against Zika virus which is highly linked with several neurological disorders.

\section{Allosteric site analysis}

The allosteric sites of ZIKV NS2B-NS3 comprise 15 residues: Asp71, Lys73, Gln74, Trp83, Leu85, Ala87, Ala88, Trp89, Gly91, Thr118, Asp120, Ile147, Leu149, Asn152 and Val155. These residues are located at topological positions to the residues of allosteric site in DENV NS2B-NS3 (Mukhametov et al., 2014). This site comprises 3 polar (Gln74, Thr118, Asn152), 9 nonpolar (Trp83, Leu85, Ala87, Ala88, Trp89, Gly91, Ile147, Leu149, Val155), 1 basic (Lys73) and 2 acidic residues (Asp71, Asp120) residues (Fig. 1). Analysis of the site suggested that it will hinder the interactions of NS3 with its cofactor NS2B, leading the inhibition of protease as reported by Shiryaev et al. (2017). Therefore, the site is used as a drug target site in this study.

\section{Structure-based virtual screening}

100,000 compounds from Mcule were docked on the allosteric site of the NS2B-NS3. Top 100 compounds were selected based on their lowest binding energy $(<$ $-9 \mathrm{kcal} / \mathrm{mol}$ ) (Table I). We analyzed different features of these selected 100 compounds, such as toxicity and Ro5 as explained by Lipinski et al. (2001). According to Lipinski et al. (2001), a good permeable drug must have these five features. First, the molecular weight of drug preferably be less than $500 \mathrm{~g} / \mathrm{mol}$; second, hydrogen bond donor (HBD) moieties in the drug be less than 5; third, hydrogen bond acceptor (HBA) be less than 10; fourth, its polar surface area (PSA) be less than $140 \AA$ and last is logarithm of partition coefficient between n-octanol and water, $\log \left(\mathrm{c}_{\text {octanol }} / \mathrm{c}_{\text {water }}\right)(\mathrm{cLog} \mathrm{P})$ must be less than five. In addition to Ro5, we determined toxicity and interactions of these compounds with NS2B-NS3. Only 14 compounds, out of the 100 selected compounds, passed Ro5 and toxicity test so they were taken for further analysis. Table I summarizes the feature of these compounds and their structures are shown in Figure 2.

Analysis of the data showed that M1 has lowest binding energy $(-9 \mathrm{kcal} / \mathrm{mol})$, suggesting its strongest interactions with the allosteric site as compared to the other 13 molecules (Table I). Such interactions are facilitated by its $6 \mathrm{HBA}$ and $1 \mathrm{HBD}$. Additionally, the cLogP and relative PSA of $\mathrm{M} 1$ falls in the allowed reign. On the other hand, M14 pose the maximum binding energy in these 14 molecules $(-7.8 \mathrm{kcal} / \mathrm{mol})$. It host $8 \mathrm{HBA}$ whereas HBA are absent in the structure.

Table I.- Features of the 14 inhibitors of the NS2B-NS3.

\begin{tabular}{|c|c|c|c|c|c|c|c|}
\hline No & Molecules & $\begin{array}{l}\text { Binding energy } \\
\text { (kcal/mol) }\end{array}$ & $\begin{array}{c}\text { Molecular weight } \\
\text { (g/mol) }\end{array}$ & cLogP & HBA & HBD & Relative PSA \\
\hline 1 & M1 & -9 & 427.55 & 3 & 6 & 1 & 0.17071 \\
\hline 2 & M2 & -8.6 & 404.322 & 4.3022 & 6 & 0 & 0.24966 \\
\hline 3 & M3 & -8.5 & 446.958 & 2.7846 & 7 & 1 & 0.22571 \\
\hline 4 & M4 & -8.4 & 375.349 & 2.1211 & 5 & 0 & 0.15715 \\
\hline 5 & M5 & -8.2 & 326.423 & 1.5393 & 5 & 0 & 0.29039 \\
\hline 6 & M6 & -8.1 & 396.493 & 1.7641 & 8 & 1 & 0.23992 \\
\hline 7 & M7 & -8.1 & 310.4 & 2.1138 & 5 & 2 & 0.23924 \\
\hline 8 & M8 & -8.1 & 441.811 & 3.8176 & 5 & 1 & 0.17553 \\
\hline 9 & M9 & -8 & 449.477 & 3.0763 & 7 & 0 & 0.21256 \\
\hline 10 & M10 & -7.9 & 333.346 & 3.0529 & 6 & 1 & 0.27512 \\
\hline 11 & M11 & -7.9 & 410.476 & 1.5397 & 9 & 0 & 0.26448 \\
\hline 12 & M12 & -7.9 & 384.459 & 3.2613 & 7 & 2 & 0.32795 \\
\hline 13 & M13 & -7.9 & 390.502 & 1.5855 & 6 & 2 & 0.25924 \\
\hline 14 & M14 & -7.8 & 355.328 & 1.6942 & 8 & 0 & 0.32856 \\
\hline
\end{tabular}

The compounds respective molecule ID's are: M1, MCULE-6483787945-0-3; M2, MCULE-2598332550-0-1; M3, MCULE-6717908673-0-1; M4, MCULE-4017669026-0-1; M-5, MCULE-4088032230-0-2; M-6, MCULE-8969381945-0-1; M7, MCULE-8631834817-0-1; M8, MCULE-3248415882-0-2; M9, MCULE-3498445429-0-1; M10, MCULE-3686500192-0-1; M11, MCULE-1165852831-0-1; M12, MCULE-7095580128-0-1; M13, MCULE-7204888140-0-1; M14, MCULE-2760593673-0-1. 


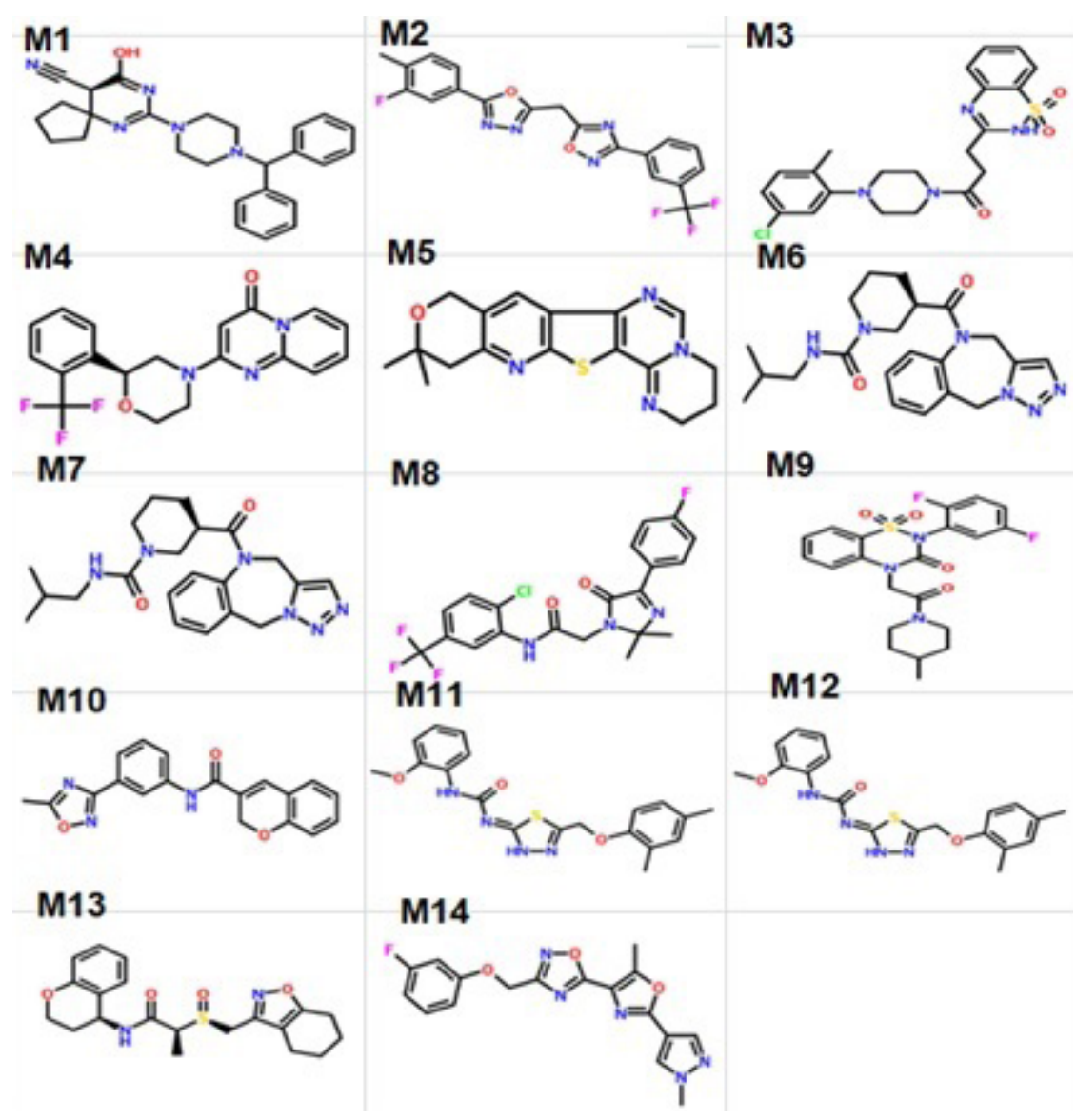

Fig. 2. Structure of selected 14 compounds.

Table II.- Features of 3 pharmacophores.

\begin{tabular}{lcccccccc}
\hline Drug ID & Fit score & Acceptors & $\begin{array}{c}\text { Binding energy } \\
\text { (kcal/mol) }\end{array}$ & Donor & Weight & Log P & TPSA & Toxicity \\
\hline ZINC00845171 & 53.33 & 7 & -8.9 & 0 & 452.44 & 3.29 & 59.73 & Nontoxic \\
ZINC08782519 & 52.88 & 6 & -8 & 2 & 413.4 & 2.93 & 75.08 & Nontoxic \\
ZINC15674546 & 52.65 & 7 & -8.3 & 2 & 432.49 & 1.61 & 96.53 & Nontoxic \\
\hline
\end{tabular}

\section{Pharmacophore}

A pharmacophore is generated by merging the chemical features of the above 14 compounds (Table I; Fig. 2). This pharmacophore host five hydrogen bond acceptors and two hydrophobic rings (Supplementary Fig. S1).

\section{Pharmacophore screening results}

The pharmacophore is used to screen 30,793 natural compounds from ZND, and 10 compounds with the maximum pharmacophore fit score (lowest 52.65) has been selected (Table II). A fit score shows the extent of similarity of one compound with the given pharmacophore. Only 3 compounds, out of these 10 compounds, passed the toxicity test. Physical features of these three compounds showed their potential to be a valuable inhibitor for the allosteric site of NS2B-NS3. For example, their HBA, $\mathrm{HBD}, \log \mathrm{P}$ and the topological polar surface area are within the allowed reign. These values are summarized in Table II. Moreover we determine the total no of hydrogen bond between these three ligands and the allosteric site (NS2B-NS3) residues (Fig. 3). Analysis of the structures showed that ZINC08782519, ZINC00845171 and ZINC15674546 forms 3, 1 and 1 hydrogen bonds, respectively, with different residues of allosteric site (Fig. 3 ). These interactions play a vital role in binding affinity of a ligand. 

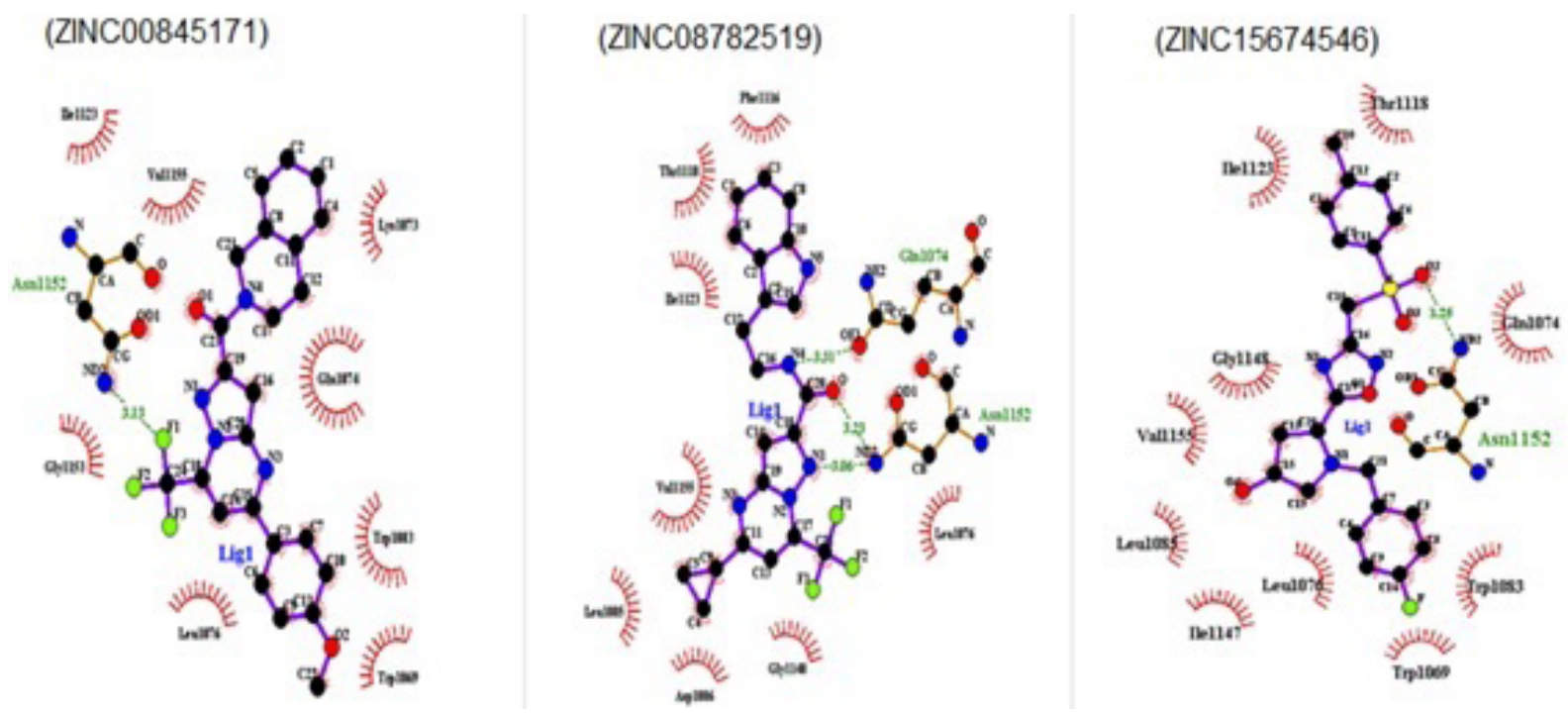

Fig. 3. Hydrogen bonding between ligands and NS2B-NS3 allosteric site residues.

\section{ADMET properties of the novel compounds}

For an orally administrated drug, it should fulfill the ADMET parameters. If the properties are weak, the candidate will have a high risk of failure. One of the compound, ZINC15674546, is eliminated from the study because it does not follow the ADMET parameters. The ADMET properties of the two novel compounds are in Table III.

Table III.- ADMET properties of two novel compounds.

\begin{tabular}{lcc}
\hline Properties & ZINC00845171 & ZINC08782519 \\
\hline $\begin{array}{l}\text { Blood-brain barrior (BBB+) } \\
\text { Human intestinal absorption } \\
\text { (HIA-) }\end{array}$ & 0.9834 & 0.9376 \\
Caco2 permeability & 1 & 1 \\
CYP450 2D6 inhibitor & 0.5 & 0.6856 \\
(non-substrate) & 0.571 & 0.7596 \\
AMES toxicity & $\mathrm{NT}$ & $\mathrm{NT}$ \\
Carcinogens & $\mathrm{NC}$ & $\mathrm{NC}$ \\
Acute oral toxicity (III) & 0.5863 & 0.5475 \\
Aquous solubility (LogS) & -3.3133 & -3.4356 \\
Rat acute toxicity & 2.8742 & 2.7787 \\
(LD50, mol/kg) & & \\
\hline
\end{tabular}

Analysis of their ADMET properties showed that both the compounds are nontoxic and noncarcinogenic as established from AEMS and carcinogenicity test (Table III). Moreover, we determined Rat Acute Toxicity LD50 for both the compounds. Another interesting feature of these compounds is optimal putative blood brain barrier value (BBB: >0.93), which is essential for penetration through the CNS. On the other hand, analysis of these structures showed these compounds are non-substrate for CYP450 2D6, suggesting a moderate metabolism rate of these compounds. Most importantly, both the compounds are water soluble as evident from their $\operatorname{logS}$ and both compounds have $\operatorname{logS}$ value $(>-3.40)$.

\section{CONCLUSION}

In silico screening against the allosteric pocket of ZIKV protease NS2B/NS3 protease led us to identify new inhibitors for the protease. Fourteen compounds have been selected after a structure-based virtual screening of 100,000 compounds, and a pharmacophore is developed. By screening available natural compounds in the ZND database against pharmcophore, top 10 compounds were selected based on their fit scores and drug properties determined. Analysis showed two (ZINC00845171 and ZINC08782519) compounds as potential inhibitors for ZIKV NS2B/NS3 protease and lead compounds against ZIKV.

\section{ACKNOWLEDGEMENT}

We gratefully acknowledge Higher Education Commission (HEC) of Pakistan for grants to this study.

\section{Supplementary material}

There is supplementary material associated with this article. Access the material online at: http://dx.doi. org/10.17582/journal.pjz/2018.50.5.1709.1715 
Statement of conflict of interest

Authors have declared no conflict of interest.

\section{REFERENCES}

Baronti, C., Piorkowski, G., Charrel, R.N., Boubis, L., Leparc-Goffart, I. and de-Lamballerie, X., 2014. Complete coding sequence of zika virus from a French polynesia outbreak in 2013. Genome Announc., 2: e00500. https://doi.org/10.1128/ genomeA.00500-14

Campos, G.S., Bandeira, A.C. and Sardi, S.I., 2015. Zika virus outbreak, Bahia, Brazil. Emerg. Infect. Dis., 21: 1885-1886. https://doi.org/10.3201/ eid2110.150847

Cheng, F., Li, W., Zhou, Y., Shen, J., Wu, Z., Liu, G., Lee, P.W. and Tang, Y., 2012. admetSAR: A comprehensive source and free tool for assessment of chemical ADMET Properties. J. Chem. Infect. Model., 52: 3099-3105. https://doi.org/10.1021/ ci300367a

Colman, E., Golden, J., Roberts, M., Egan, A., Weaver, J. and Rosebraugh, C., 2012. The FDA's assessment of two drugs for chronic weight management. N. Engl. J. Med., 367: 1577-1579. https://doi. org/10.1056/NEJMp1211277

Dick, G.W.A., Kitchen, S.F. and Haddow, A.J., 1952. Zika virus (I). Isolations and serological specificity. Trans. R. Soc. trop. Med. Hyg., 46: 509-520. https:// doi.org/10.1016/0035-9203(52)90042-4

Driggers, R.W., Ho, C.Y., Korhonen, E.M., Kuivanen, S., Jääskeläinen, A.J., Smura, T., Rosenberg, A., Hill, D.A., de Biasi, R.L., Vezina, G., Timofeev, J., Rodriguez, F.J., Levanov, L., Razak, J., Iyengar, P., Hennenfent, A., Kennedy, R., Lanciotti, R., du Plessis, A. and Vapalahti, O., 2016. Zika virus infection with prolonged maternal viremia and fetal brain abnormalities. N. Engl. J. Med., 374: 21422151. https://doi.org/10.1056/NEJMoa1601824

Gruba, N., Rodriguez-Martinez, J.I., Grzywa, R., Wysocka, M., Skoreński, M., Burmistrz, M., Lecka, M., Lesner, A., Sienczyk, M. and Pyrć, K., 2016. Substrate profiling of Zika virus NS2B-NS3 protease. FEBS Lett., 590: 3459-3468. https://doi. org/10.1002/1873-3468.12443

Ikejezie, J., Shapiro, C.N., Kim, J., Chiu, M., Almiron, M., Ugarte, C., Espinal, M.A. and Aldighieri, S., 2016. Zika virus transmission-Region of the Americas. MMWR Morb. Mort., 66: 329-334. https://doi.org/10.15585/mmwr.mm6612a4

Jamil, F., Raheel, S. and Rasheed, H., 2018. Docking of smaller ligands in the heme pocket of Hell's Gate
Globin IV. Pakistan J. Zool., 4: 1199-1600 http:// dx.doi.org/10.17582/journal.pjz/2018.50.4.sc9

Kiss, R., Sandor, M. and Szalai, F.A., 2012. A public web service for drug discovery. J. Cheminform., 4: 17. http://mcule.com

Kuno, G. and Chang, G.J., 2007. Full-length sequencing and genomic characterization of Bagaza Kedougou and Zika viruses. Arch. Virol., 152: 687-696. https:// doi.org/10.1007/s00705-006-0903-z

Lei, J., Hansen, G., Nitsche, C., Klein, C.D., Zhang, L. and Hilgenfeld, R., 2016. Crystal structure of Zika virus NS2B-NS3 protease in complex with a boronate inhibitor. Science, 353: 503-505. https:// doi.org/10.1126/science.aag2419

Li, Y., Zhang, Z., Phoo, W.W., Loh, Y.R., Wang, W., Liu, S., Chen, M.W., Hung, A.W., Keller, T.H., Luo, D. and Kang, C., 2017. Structural dynamics of Zika virus NS2B-NS3 protease binding to dipeptide inhibitors. Structure, 25: 1242-1250. https://doi. org/10.1016/j.str.2017.06.006

Lill, M.A. and Danielson, M.L., 2011. Computer-aided drug design platform using PyMOL. J. Comput. Aided Mol. Des., 25: 13-19. https://doi.org/10.1007/ s10822-010-9395-8

Lipinski, C.A., Lombardo, F., Dominy, B.W. and Feeney, P.J., 2001. Experimental and computational approaches to estimate solubility and permeability in drug discovery and development settings. $A d v$. Drug Deliv. Rev., 46: 3-26. https://doi.org/10.1016/ S0169-409X(00)00129-0

Mackenzie, J., 2005. Wrapping things up about virus RNA replication. Traffic, 6: 967-977. https://doi. org/10.1111/j.1600-0854.2005.00339.x

Moghadam, S.R.J., Bayrami, S., Moghadam, S.J., Golrokhi, R., Pahlaviani, F.G. and Seyedalinaghi, S.A.S., 2016. Zika virus: A review of literature. Asian Pac. J. trop. Med., 6: 989-994. https://doi. org/10.1016/j.apjtb.2016.09.007

Mukhametov, A., Newhouse, E.I., Aziz, N.A., Saito, J.A. and Alam, M., 2014. Allosteric pocket of the dengue virus (serotype 2) NS2B/NS3 protease: In silico ligand screening and molecular dynamics studies of inhibition. J. Mol. Graph. Model., 52: 103-113. https://doi.org/10.1016/j.jmgm.2014.06.008

Nowakowski, T.J., Pollen, A.A., di Lullo, E., SandovalEspinosa, C., Bershteyn, M. and Kriegstein, A.R., 2016. Expression analysis highlights AXL as a candidate zika virus entry receptor in neural stem cells. Cell Stem Cell, 18: 591-596. https://doi. org/10.1016/j.stem.2016.03.012

Rana, N., Ehsan, N., Ihsan, A. and Jamil, F., 2017. Analyses of the pseudogenes of Helicobacter 
pylori. Pakistan J. Zool., 49: 1261-1265. https:// doi.org/10.17582/journal.pjz/2017.49.4.1261.1265 Shiryaev, S.A., Farhy, C., Pinto, A., Huang, C.T., Simonetti, N., Elong-Ngono, A., Dewing, A., Shresta, S., Pinkerton, A.B., Cieplak, P., Strongin, A.Y. and Terskikh, A.V., 2017. Characterization of the Zika virus two-component NS2B-NS3 protease and structure-assisted identification of allosteric small-molecule antagonists. Antivir. Res., 143: 218-229. https://doi.org/10.1016/j. antiviral.2017.04.015

Sironi, M., Forni, D., Clerici, M. and Cagliani, R., 2016. Nonstructural proteins are preferential positive selection targets in Zika virus and related Flaviviruses. PLoS Negl. Trop. Dis., 10: e0004978. https://doi.org/10.1371/journal.pntd.0004978

Tan, C.W., Sam, I.C., Chong, W.L., Lee, V.S. and Chan, Y.F., 2017. Polysulfonate suramin inhibits Zika virus infection. Antivir. Res., 143: 186-194. https:// doi.org/10.1016/j.antiviral.2017.04.017

Tariq, F., Khalid, Q., Sehgal, S.A., Mannan, S. and
Jamil, F., 2016. Possible roles of the pseudogenes of Salmonella typhimurium. Pakistan J. Zool., 48: 1805-1810.

Trott, O. and Olson, A.J., 2010. AutoDock vina: improving the speed and accuracy of docking with a new scoring function, efficient optimization, and multithreading. J. Comput. Chem., 31: 455-461.

Wallace, A.C., Laskowski, R.A. and Thornton, J.M., 1995. LIGPLOT: A program to generate schematic diagrams of protein-ligand interactions. Protein Eng., 8: 127-134. https://doi.org/10.1093/ protein/8.2.127

Wolber, G. and Langer, T., 2005. LigandScout: 3-D pharmacophores derived from protein-bound ligands and their use as virtual screening filters. J. Chem. Infect. Model., 45: 160-169. https://doi. org/10.1021/ci049885e

Yildiz, M., Ghosh, S., Bell, J.A., Sherman, W. and Hardy, J.A., 2013. Allosteric inhibition of the NS2B-NS3 protease from dengue virus. ACS Chem. Biol., 8: 2744-2752. https://doi.org/10.1021/cb400612h 